\title{
Putting $\mathrm{M}$ theory on a computer
}

\author{
Jun Nishimura* \\ High Energy Accelerator Research Organization (KEK), Tsukuba 305-0801, Japan, \\ and Graduate University for Advanced Studies (SOKENDAI), Tsukuba 305-0801, Japan \\ E-mail: jnishi@post.kek.jp
}

\section{Konstantinos N. Anagnostopoulos}

National Technical University of Athens, Zografou Campus, GR-15780 Athens, Greece E-mail: konstantemail.ntua.gr

\section{Masanori Hanada}

Theoretical Physics Laboratory, RIKEN Nishina Center,

2-1 Hirosawa, Wako, Saitama 351-0198, Japan

E-mail: hana@riken.jp

\section{Shingo Takeuchi}

Graduate University for Advanced Studies (SOKENDAI), Tsukuba 305-0801, Japan

E-mail: shingo@post.kek.jp

\begin{abstract}
We propose a non-lattice simulation for studying supersymmetric matrix quantum mechanics in a non-perturbative manner. In particular, our method enables us to put $\mathrm{M}$ theory on a computer based on its matrix formulation proposed by Banks, Fischler, Shenker and Susskind. Here we present Monte Carlo results of the same matrix model but in a different parameter region, which corresponds to the 't Hooft large- $N$ limit at finite temperature. In the strong coupling limit the model has a dual description in terms of the $N$ D0-brane solution in 10d type IIA supergravity. Our results provide highly nontrivial evidences for the conjectured duality. In particular, the energy (and hence the entropy) of the non-extremal black hole has been reproduced by solving directly the strongly coupled dynamics of the D0-brane effective theory.
\end{abstract}

The XXV International Symposium on Lattice Field Theory

July 30 - August 42007

Regensburg, Germany

\footnotetext{
* Speaker.
} 


\section{Introduction}

Large- $N$ gauge theories are playing more and more important roles in theoretical particle physics. In particular, they are considered to be useful in formulating superstring/M theories nonperturbatively extending the idea of matrix models, which was successful for string theories in sub-critical dimensions. For instance, it has been conjectured that critical string/M theories can be formulated in terms of matrix models, which can be formally obtained by dimensionally reducing $\mathrm{U}(N)$ super Yang-Mills theory in ten dimensions to $D=0,1,2$ dimensions. The $D=1$ case corresponds to the Matrix theory [1], which is conjectured to describe M Theory microscopically.

Another important conjecture, which has been studied intensively over the decade, concerns the duality between the strongly coupled large- $N$ gauge theory and the weakly coupled supergravity. The best understood example is the AdS/CFT correspondence, but there are numerous extensions to non-conformal field theories as well. In particular, large- $N$ gauge theories in low dimensions have been studied intensively at finite temperature, which revealed intriguing connections to the black-hole thermodynamics [2, 3, 4, 5].

Monte Carlo simulation of large- $N$ gauge theories is expected to be very useful in order to confirm these conjectures or to make use of them. Indeed, the totally reduced models [6] (the gauge theory reduced to $D=0$ dimension) have been studied in refs. [७] [7]. In the $D \geq 1$ case, some sort of "discretization" is needed in order to put the theory on a computer. However, lattice simulation of supersymmetric gauge theories is not straightforward. In some cases the lack of manifest supersymmetry just necessitates cumbersome fine-tuning, but in the worse cases the chiral and/or Majorana nature of fermions makes it difficult to even formulate an appropriate lattice theory. We propose to circumvent all these problems inherent in the lattice approach by adopting a non-lattice approach [9] for one-dimensional supersymmetric gauge theories. This approach, in particular, enables us to put $\mathrm{M}$ theory on a computer using the Matrix theory [1], which takes the form of a 1d $\mathrm{U}(N)$ gauge theory with 16 supercharges.

Here we demonstrate our approach by studying the same model but in a different parameter region, which corresponds to the 't Hooft large- $N$ limit at finite temperature [10]. In the strong coupling limit the model has a dual description [2] in terms of the $N$ D0-brane solution in type IIA supergravity. Our results provide highly nontrivial evidences for the conjectured duality. In particular, the energy (and hence the entropy) of the non-extremal black hole has been reproduced by solving directly the strongly coupled dynamics of the D0-brane effective theory.

\section{SUSY matrix quantum mechanics with 16 supercharges}

The model can be obtained formally by dimensionally reducing $10 \mathrm{~d} \mathscr{N}=1$ super Yang-Mills theory to $1 \mathrm{~d}$. The action is given by

$$
S=\frac{1}{g^{2}} \int_{0}^{\beta} d t \operatorname{tr}\left\{\frac{1}{2}\left(D_{t} X_{i}\right)^{2}-\frac{1}{4}\left[X_{i}, X_{j}\right]^{2}+\frac{1}{2} \psi_{\alpha} D_{t} \psi_{\alpha}-\frac{1}{2} \psi_{\alpha}\left(\gamma_{i}\right)_{\alpha \beta}\left[X_{i}, \psi_{\beta}\right]\right\}
$$

where $D_{t}=\partial_{t}-i[A(t), \cdot]$ represents the covariant derivative with the gauge field $A(t)$ being an $N \times N$ Hermitian matrix. This model can be viewed as a one-dimensional $\mathrm{U}(N)$ gauge theory with adjoint matters. The bosonic matrices $X_{i}(t)(i=1, \cdots, 9)$ come from spatial components of the 
10d gauge field, while the fermionic matrices $\psi_{\alpha}(t)(\alpha=1, \cdots, 16)$ come from a Majorana-Weyl spinor in 10d. The $16 \times 16$ matrices $\gamma_{i}$ in 2.1) act on spinor indices and satisfy the Euclidean Clifford algebra $\left\{\gamma_{i}, \gamma_{j}\right\}=2 \delta_{i j}$. We impose periodic and anti-periodic boundary conditions on the bosons and fermions, respectively. The extent $\beta$ in the Euclidean time direction then corresponds to the inverse temperature $\beta \equiv 1 / T$. The 't Hooft coupling constant is given by $\lambda \equiv g^{2} N$, and the dimensionless effective coupling constant is given by $\tilde{\lambda}=\lambda / T^{3}$. Without loss of generality we set $\lambda=1$, hence low (high) $T$ corresponds to strong (weak) coupling strength, respectively.

\section{Non-lattice simulation for SUSY matrix quantum mechanics}

We fix the gauge by the static diagonal gauge

$$
A(t)=\frac{1}{\beta} \operatorname{diag}\left(\alpha_{1}, \cdots \alpha_{N}\right),
$$

where $\alpha_{a}$ can be chosen to satisfy the constraint $\max _{a}\left(\alpha_{a}\right)-\min _{a}\left(\alpha_{a}\right) \leq 2 \pi$ using the large gauge transformation. We have to add to the action a term $S_{\mathrm{FP}}=-\sum_{a<b} 2 \ln \left|\sin \frac{\alpha_{a}-\alpha_{b}}{2}\right|$, which appears from the Faddeev-Popov procedure.

We make a Fourier expansion

$$
X_{i}^{a b}(t)=\sum_{n=-\Lambda}^{\Lambda} \tilde{X}_{i n}^{a b} \mathrm{e}^{i \omega n t} ; \psi_{\alpha}^{a b}(t)=\sum_{r=-\Lambda^{\prime}}^{\Lambda^{\prime}} \tilde{\psi}_{\alpha r}^{a b} \mathrm{e}^{i \omega r t},
$$

where $\omega=\frac{2 \pi}{\beta}$ and $\Lambda^{\prime} \equiv \Lambda-1 / 2$. The indices $n$ and $r$ take integer and half-integer values, respectively, corresponding to the imposed boundary conditions. Introducing a shorthand notation

$$
\left(f^{(1)} \cdots f^{(p)}\right)_{n} \equiv \sum_{k_{1}+\cdots+k_{p}=n} f_{k_{1}}^{(1)} \cdots f_{k_{p}}^{(p)}
$$

we can write the action (2.1) as $S=S_{\mathrm{b}}+S_{\mathrm{f}}$, where

$$
\begin{aligned}
& S_{\mathrm{b}}=N \beta\left[\frac{1}{2} \sum_{n=-\Lambda}^{\Lambda}\left(n \omega-\frac{\alpha_{a}-\alpha_{b}}{\beta}\right)^{2} \tilde{X}_{i,-n}^{b a} \tilde{X}_{i n}^{a b}-\frac{1}{4} \operatorname{tr}\left(\left[\tilde{X}_{i}, \tilde{X}_{j}\right]^{2}\right)_{0}\right] \\
& S_{\mathrm{f}}=\frac{1}{2} N \beta \sum_{r=-\Lambda^{\prime}}^{\Lambda^{\prime}}\left[i\left(r \omega-\frac{\alpha_{a}-\alpha_{b}}{\beta}\right) \tilde{\Psi}_{\alpha r}^{b a} \tilde{\psi}_{\alpha r}^{a b}-\left(\gamma_{i}\right)_{\alpha \beta} \operatorname{tr}\left\{\tilde{\Psi}_{\alpha r}\left(\left[\tilde{X}_{i}, \tilde{\psi}_{\beta}\right]\right)_{r}\right\}\right] .
\end{aligned}
$$

It is important that we have introduced the cutoff $\Lambda$ after fixing the gauge non-perturbatively. This is possible only in $1 \mathrm{~d}$. In higher dimensions, the momentum cutoff regularization inevitably breaks the gauge invariance. In the bosonic case, we have checked explicitly [7] that the results of the non-lattice simulation agree with the results of the lattice simulation in the continuum limit.

Note that our action is nothing but the gauge-fixed action in the continuum except for having a Fourier mode cutoff. This leads to various advantages over the lattice approach proposed in ref. [11]. Supersymmetry, which is mildly broken by the cutoff, is shown (in 1d Wess-Zumino model) to be restored much faster than the continuum limit is achieved. In fact, the continuum limit is also approached faster than one would naively expect from the number of degrees of freedom. These are understandable from the fact that the modes above the cutoff are naturally suppressed by the kinetic 
term. A further (albeit technical) advantage of our formulation is that the Fourier acceleration, which eliminates the critical slowing down completely [12], can be implemented without extra cost since we are dealing with Fourier modes directly. We consider that all these merits of the present approach compensate the superficial increase in the computational effort by the factor of $\mathrm{O}(\Lambda)$ compared to the lattice approach [11] with the same number of degrees of freedom.

The fermionic action $S_{\mathrm{f}}$ may be written in the form $S_{\mathrm{f}}=\frac{1}{2} \mathscr{M}_{A \alpha r ; B \beta s} \tilde{\psi}_{\alpha r}^{A} \tilde{\psi}_{\beta s}^{B}$, where we have expanded $\tilde{\psi}_{\alpha r}=\sum_{A=1}^{N^{2}} \tilde{\psi}_{\alpha r}^{A} t^{A}$ in terms of $\mathrm{U}(N)$ generators $t^{A}$. Integrating out the fermions, we obtain the Pfaffian $\operatorname{Pf} \mathscr{M}$, which is complex for generic configurations of the remaining bosonic variables. However, it turns out to be real positive with high accuracy in the temperature region studied in the present work. Hence we can replace it by $|\operatorname{Pf} \mathscr{M}|=\operatorname{det}\left(\mathscr{D}^{1 / 4}\right)$, where $\mathscr{D}=\mathscr{M}^{\dagger} \mathscr{M}$. One can then apply the Rational Hybrid Monte Carlo algorithm [13] to study the system in an efficient way.

\section{Results}

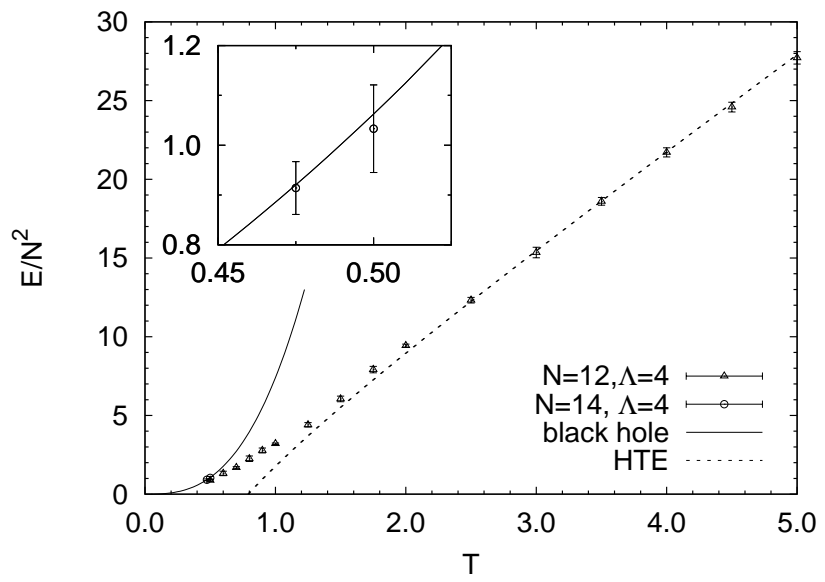

Figure 1: The energy (normalized by $N^{2}$ ) is plotted against $T$. The dashed line represents the result obtained by HTE up to the next leading order for $N=12$ [14]. The solid line represents the asymptotic power-law behavior at small $T$ predicted by the gauge/gravity duality. The upper left panel zooms up the region, where the power-law behavior sets in.

In fig. 1 we plot the internal energy defined by $E=\frac{\partial}{\partial \beta}(\beta \mathscr{F})$, where $\mathscr{F}$ is the free energy of the system. Our results interpolate nicely the weak coupling behavior - calculated by the high temperature expansion (HTE) up to the next leading order [14] - and the strong coupling behavior $\frac{E}{N^{2}}=7.4 \cdot T^{2.8}$ predicted by the gauge/gravity duality [2] from the dual black-hole geometry [15]. The power-law behavior sets in at $T \simeq 0.5$, which is reasonable since the effective coupling constant is given by $\tilde{\lambda}=1 / T^{3}$ in our convention.

In ref. [ $₫$ the Gaussian expansion method was applied to the present model, and the energy obtained at the leading order was fitted nicely to the power law $E / N^{2}=3.4 \cdot T^{2.7}$ within $0.25 \lesssim T \lesssim$ 1. This result is in reasonable agreement with our data at $T \sim 1$, but disagrees at lower temperature.

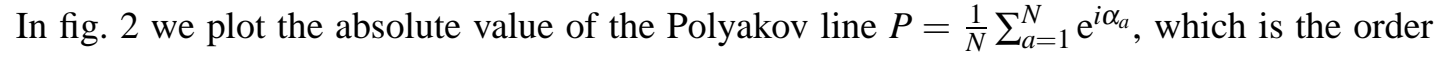
parameter for the SSB of the U(1) symmetry. It changes smoothly for the range of $T$ investigated, 


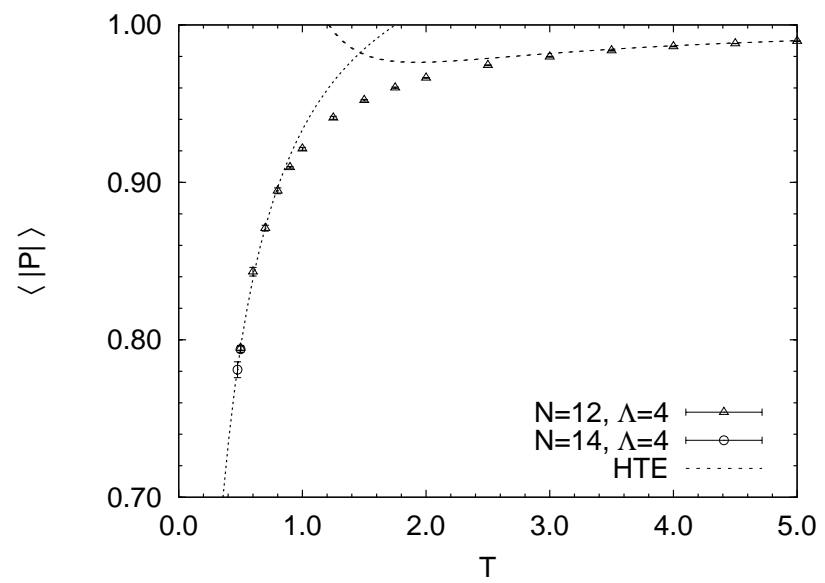

Figure 2: The Polyakov line is plotted against $T$. The dashed line represents the result of HTE up to the next leading order for $N=12$ [14]. The dotted line represents a fit to eq. 4.1] with $a=0.15$ and $b=0.072$.

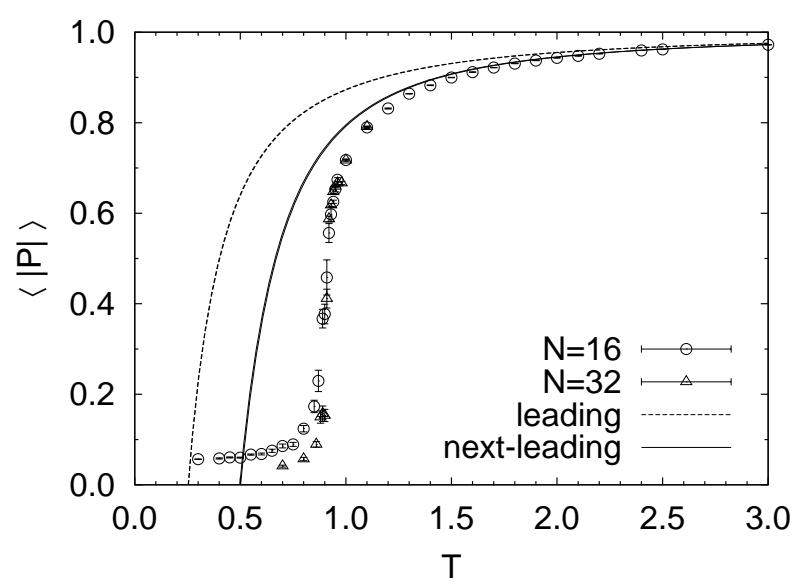

Figure 3: The Polyakov line for the bosonic model [16]. The results obtained by HTE for $N=16$ at the leading order (dashed line) and up to the next leading order (solid line) [14] are also plotted.

which implies the absence of a phase transition as predicted by the gauge/gravity duality [3, 5]. At low $T$ it can be fitted nicely to the asymptotic behavior characteristic to a deconfined theory:

$$
\langle|P|\rangle=\exp \left(-\frac{a}{T}+b\right)
$$

This is in striking contrast to the bosonic case [16] shown in fig. 3 for comparison ${ }^{1}$.

\footnotetext{
${ }^{1}$ In ref. [16] it was found that there are actually three phases in the bosonic model. The intermediate phase appears in a very narrow range of temperature $T_{\mathrm{c} 2}<T<T_{\mathrm{c} 1}$, where $T_{\mathrm{c} 1}=0.905(2)$ and $T_{\mathrm{c} 2}=0.8761(3)$, and it is characterized by the non-uniform eigenvalue distribution of the holonomy matrix. The order of phase transitions are second order at $T=T_{\mathrm{c} 1}$, and third order at $T=T_{\mathrm{c} 2}$.
} 


\section{Summary and future prospects}

We have presented the first Monte Carlo results for the maximally supersymmetric matrix quantum mechanics. The non-lattice simulation enabled us to study the low temperature behavior, which was not accessible by HTE. This provided highly non-trivial evidences for the gauge/gravity duality. In particular, we observed that the internal energy asymptotes nicely at low temperature to the result obtained from the dual black-hole geometry.

Our results suggest that not only the power but also the coefficient of the power-law behavior is reproduced correctly by the gauge theory in the $N \rightarrow \infty$ and $\tilde{\lambda} \rightarrow \infty$ limits. This implies that we were able to identify the microscopic degrees of freedom, which accounts for the BekensteinHawking entropy for the 10d non-extremal black hole. They are nothing but the open strings attached to the D0-branes, which are described by the gauge theory. Note that this conclusion is much stronger than that of Strominger-Vafa [17], which deals with extremal black holes and relies on the supersymmetric non-renormalization theorem.

Assuming the duality to hold in the stronger sense, one may go on and investigate the quantum and stringy corrections to the black-hole thermodynamics from the gauge theory side as finite- $N$ and finite- $\tilde{\lambda}$ effects. In particular it would be interesting to understand the physical meaning of the infrared instability observed in our simulation [10] from that perspective.

When we simulate $\mathrm{M}$ theory, we should impose periodic boundary conditions on fermions, and then the system with finite $N$ corresponds to a sector of M theory compactified on a light-like circle [18]. However, the Pfaffian will not be close to real positive due to the fermionic zero modes unlike the situation in the present work. That may cause a technical problem known as the sign problem when one tries to investigate the large- $N$ behavior. The same problem occurs in Monte Carlo studies of the totally reduced models, in which the phase of the Pfaffian is speculated [19] to induce the spontaneous breaking of $\mathrm{SO}(10)$ symmetry down to $\mathrm{SO}(4)$. This pattern of SSB is indeed suggested by the Gaussian expansion method [20], and it provides a natural scenario for the dynamical generation of $4 \mathrm{~d}$ space-time [21]. In ref. [22] it was conjectured, based on the Eguchi-Kawai equivalence, that a similar phenomenon occurs also in the supersymmetric matrix quantum mechanics. We hope to address such an issue from first principles by using the non-lattice simulation method together with the idea proposed in ref. [8] to overcome the sign problem.

\section{References}

[1] T. Banks, W. Fischler, S. H. Shenker and L. Susskind, M theory as a matrix model: A conjecture, Phys. Rev. D 55 (1997) 5112 [hep-th/9610043].

[2] N. Itzhaki, J. M. Maldacena, J. Sonnenschein and S. Yankielowicz, Supergravity and the large N limit of theories with sixteen supercharges, Phys. Rev. D 58 (1998) 046004 [hep-th/9802042].

[3] J. L. F. Barbon, I. I. Kogan and E. Rabinovici, On stringy thresholds in SYM/AdS thermodynamics, Nucl. Phys. B 544 (1999) 104 [hep-th/9809033].

[4] D. Kabat, G. Lifschytz and D. A. Lowe, Black hole thermodynamics from calculations in strongly coupled gauge theory, Phys. Rev. Lett. 86 (2001) 1426 [hep-th/ 0007051 ]; Black hole entropy from non-perturbative gauge theory, Phys. Rev. D 64 (2001) 124015 [hep-th/ 0105171$].$ 
[5] O. Aharony, J. Marsano, S. Minwalla, K. Papadodimas, M. Van Raamsdonk and T. Wiseman, The phase structure of low dimensional large $N$ gauge theories on tori, JHEP 0601 (2006) 140 [hep-th/0508077].

[6] N. Ishibashi, H. Kawai, Y. Kitazawa and A. Tsuchiya, A large-N reduced model as superstring, Nucl. Phys. B 498 (1997) 467 [hep-th/9612115].

[7] W. Krauth, H. Nicolai and M. Staudacher, Monte Carlo approach to M-theory, Phys. Lett. B 431 (1998) 31 [hep-th/9803117]; J. Ambjorn, K. N. Anagnostopoulos, W. Bietenholz, T. Hotta and J. Nishimura, Monte Carlo studies of the IIB matrix model at large N, JHEP 07 (2000) 011 [hep-th/0005147].

[8] K. N. Anagnostopoulos and J. Nishimura, New approach to the complex-action problem and its application to a nonperturbative study of superstring theory, Phys. Rev. D 66 (2002) 106008 [hep-th/0108041].

[9] M. Hanada, J. Nishimura and S. Takeuchi, Non-lattice simulation for supersymmetric gauge theories in one dimension, arXiv:0706.1647 [hep-lat], to appear in Phys. Rev. Lett.

[10] K. N. Anagnostopoulos, M. Hanada, J. Nishimura and S. Takeuchi, Monte Carlo studies of supersymmetric matrix quantum mechanics with sixteen supercharges at finite temperature, arXiv:0707.4454 [hep-th].

[11] S. Catterall and T. Wiseman, Towards lattice simulation of the gauge theory duals to black holes and hot strings, arXiv:0706.3518 [hep-lat]; See also S. Catterall's contribution to this volume.

[12] S. Catterall and S. Karamov, Testing a Fourier accelerated hybrid Monte Carlo algorithm, Phys. Lett. B 528 (2002) 301 [hep-lat/0112025].

[13] M. A. Clark, A. D. Kennedy and Z. Sroczynski, Exact 2+1 flavour RHMC simulations, Nucl. Phys. Proc. Suppl. 140 (2005) 835 [hep-lat/ 0409133 ].

[14] N. Kawahara, J. Nishimura and S. Takeuchi, in preparation.

[15] I.R. Klebanov and A.A. Tseytlin, Entropy of near-extremal black p-branes, Nucl. Phys. B 475 (1996) 164 [hep-th/9604089].

[16] N. Kawahara, J. Nishimura and S. Takeuchi, Phase structure of matrix quantum mechanics at finite temperature, arXiv:0706.3517 [hep-th].

[17] A. Strominger and C. Vafa, Microscopic origin of the Bekenstein-Hawking entropy, Phys. Lett. B 379 (1996) 99 [hep-th/9601029].

[18] N. Seiberg, Why is the matrix model correct?, Phys. Rev. Lett. 79 (1997) 3577 [hep-th/9710009].

[19] J. Nishimura and G. Vernizzi, Spontaneous breakdown of Lorentz invariance in IIB matrix model, JHEP 04 (2000) 015 [hep-th / 00 03223]; Brane world generated dynamically from string type IIB matrices, Phys. Rev. Lett. 85 (2000) 4664 [hep-th/ 0007022$].$

[20] J. Nishimura and F. Sugino, Dynamical generation of four-dimensional space-time in the IIB matrix model, JHEP 05 (2002) 001 [hep-th/ 0111102 ]; T. Aoyama and H. Kawai, Higher order terms of improved mean field approximation for IIB matrix model and emergence of four-dimensional space-time, Prog. Theor. Phys. 116 (2006) 405 [hep-th/ 0603146$].$

[21] H. Aoki, S. Iso, H. Kawai, Y. Kitazawa and T. Tada, Space-time structures from IIB matrix model, Prog. Theor. Phys. 99 (1999) 713 [hep-th/9802085].

[22] N. Kawahara and J. Nishimura, The large $N$ reduction in matrix quantum mechanics: A bridge between BFSS and IKKT, JHEP 09 (2005) 040 [hep-th/0505178]. 